\title{
Ubiquitin ligase Siah1 promotes the migration and invasion of human glioma cells by regulating HIF-1 $\alpha$ signaling under hypoxia
}

\author{
HENGLIANG SHI ${ }^{1,2^{*}}$, BAO ZHENG $^{2 *}$, YUXUAN WU $^{2}$, YUAN TANG $^{2}$, LEI WANG $^{1}$, \\ YONG GAO $^{2}$, HUI GONG ${ }^{2}$, JIN DU ${ }^{2}$ and RUTONG YU ${ }^{1,2}$ \\ ${ }^{1}$ Department of Neurosurgery, Affiliated Hospital of Xuzhou Medical College, Xuzhou, Jiangsu; \\ ${ }^{2}$ The Graduate School, Xuzhou Medical College, Xuzhou, Jiangsu, P.R. China
}

Received October 13, 2014; Accepted December 5, 2014

DOI: $10.3892 /$ or.2014.3695

\begin{abstract}
It has been reported that by regulating PHD3 stability, Siah1 contributes to the abundance of hypoxiainducible factor (HIF)-1 $\alpha$, thereby playing an important role in the cellular response to hypoxia. However, the expression level and functional significance of Siah1 in human malignant glioma, which is characterized by high migration and invasion potential, have never been investigated. We report here, that Siah1 was expressed highly in human glioma tissues compared with its expression in normal brain tissues and was correlated with advanced tumor status and stage. The knockdown of Siah1 by short-hairpin RNA severely suppressed the migration and invasion of human glioma U251 cells under hypoxia, while overexpression of Siah1 promoted it. Furthermore, we demonstrated that the glioma cell migration and invasion under hypoxia mediated by Siah1 was achieved by reducing the stability of PHD3, which protected the HIF-1 $\alpha$ from degradation. These findings suggest that Siah1 plays important roles in the migration and invasion of human glioma cells under hypoxia, which may provide some guidance for the targeted therapy of human glioma based on the interference of the Siah1-PHD3-HIF-1 $\alpha$ signaling pathway.
\end{abstract}

\section{Introduction}

Glioblastoma multiforme (GBM) is the most common and malignant primary tumor that develops in the central nervous system. Despite advances in surgery and adjuvant therapy, the overall 5-year survival rate of GBM remains less than 5\% and is even worse for elderly patients due to the highly invasive ability of these cancer cells $(1,2)$.

Correspondence to: Dr Rutong Yu, Department of Neurosurgery, Affiliated Hospital of Xuzhou Medical College, 99 West Huaihai Road, Xuzhou, Jiangsu 221002, P.R. China

E-mail: yu.rutong@163.com

${ }^{*}$ Contributed equally

Key words: Siah1, PHD3, HIF-1 $\alpha$, hypoxia, glioma
Hypoxia-inducible factor $1 \alpha(\mathrm{HIF}-1 \alpha)$ and hypoxia-responsive genes play important roles in glioma migration and invasion (3-5). Hypoxia is important in the biology and aggression of human glial brain tumors $(6,7)$. Although HIF-1 $\alpha$ is maintained at a low level in normoxic cells due to the degradation mediated by the proteasome (8), it plays a critical role in the cellular response to tumor hypoxia, which may be a major obstacle to the killing of cancer cells by radiotherapy and chemotherapy (6). It has been reported that HIF-1 $\alpha$ is upregulated in a variety of human cancers, including prostate (9), breast (10) and glioma (3). Inhibition of HIF-1 $\alpha$ expression with antisense oligonucleotides was found to decrease the survival of glioblastoma cells and induce p53-independent apoptosis (11). Moreover, silencing of HIF-1 $\alpha$ by RNA interference reduced human glioma migration and invasion $(12,13)$. Although the function of HIF-1 $\alpha$ has been widely studied under hypoxic stress, the mechanism regulating the activation of HIF-1 $\alpha$ is still unclear, and should be further understood for designing more effective therapeutic strategies.

The protein seven in absentia (SINA) was firstly discovered in R7 cells, and functions in the cell fate in the Drosophila eye (14). Two homologs of the SINA gene have been identified in humans, Siah1 and Siah2 (15). Siah1 and Siah2 have been shown to dominate the stability of several substrates, including nuclear corepressor, $\beta$-catenin, TRAF2, $\alpha$-ketoglutarate dehydrogenase and prolyl hydroxylase 3 (PHD3), thereby affecting diverse cellular processes, such as signaling, survival and mitochondrial biogenesis (16-20). In addition to the physiological function, Siah1 is found to be involved in several types of cancer. It has been reported that Siah1 plays a role as a tumor suppressor in hepatocellular carcinomas and breast cancer $(21,22)$. However, in nasopharyngeal carcinoma, Siah1 has been reported as an oncogene and is significantly correlated with advanced tumor status and stage (23). To date, the role of Siah1 in human glioma has not been elucidated.

In the present study, we investigated the role and intracellular signaling pathway of Siah1 in the aggressive behavior of glioma under hypoxic stress. We found that Siah1 levels in glioma tissues were significantly upregulated, suggesting a potential role of this protein in the progression of gliomas. Then, we observed that the knockdown of Siah1 inhibited the migration and invasion of human glioma cells under hypoxia, while overexpression of Siah1 promoted it. Furthermore, we 
demonstrated that the Siah1-regulated migration and invasion of human glioma cells under hypoxia were mediated by decreasing the stability of PHD3, thereby stabilizing the HIF-1 $\alpha$.

\section{Materials and methods}

Tissue samples. Six specimens of non-tumorous brain tissues (internal decompression in cerebral trauma) and 16 specimens of glioma tissues were obtained from the Affiliated Hospital of Xuzhou Medical College (Xuzhou, China). Surgically removed tissues were sampled for histological diagnosis, and the remaining tissues were immediately frozen in liquid nitrogen and stored at $-80^{\circ} \mathrm{C}$. The present study was approved by the Research Ethics Committee of Xuzhou Medical College, and informed consent was provided by the patients.

Cell culture. Cell lines (293T and U251) were purchased from the Cell Bank of the Shanghai Institutes of the Chinese Academy of Sciences and were cultured in Dulbecco's modified Eagle's medium (DMEM; Invitrogen, Carlsbad, CA, USA) supplemented with $10 \%$ fetal bovine serum (FBS; Evergreen Biological Engineering Co., Hangzhou, China) at $37^{\circ} \mathrm{C}$ in $5 \%$ $\mathrm{CO}_{2}$. For hypoxia, the cells were cultured in a modular incubator chamber (150i; Thermo Fisher Scientific, Waltham, MA, USA) at $37^{\circ} \mathrm{C}$ with $5 \% \mathrm{CO}_{2}, 1 \% \mathrm{O}_{2}$ and $94 \% \mathrm{~N}_{2}$.

Constructs and production of the lentivirus. For overexpression of Siah1, the Siah1 cDNA was inserted into the pWPXLd plasmid using BamHI and MluI sites. For silencing of Siah1, two short hairpin RNA (shRNA) duplexes were designed as follows: shSiah1A F, TGATAGGAACACGCAAGCAATT CAAGAGATTGCTTGCGTGTTCCTATCTTTTTTC and shSiah1A R, TCGAGAAAAAAGATAGGAACACGCAAGC AATCTCTTGAATTGCTTGCGTGTTCCTATCA; shSiah1B F, TCACACCTTTGAGCTTAATCTTCAAGAGAGATTAA GCTCAAAGGTGTGTTTTTTC and shSiah1B R, TCGAGA AAAAACACACCTTTGAGCTTAATCTCTCTTGAAGAT TAAGCTCAAAGGTGTGA. The shRNA oligomers and nontargeting oligomers (scramble) were annealed and then subcloned into the pLL3.7 plasmid by the HpaI and XhoI cloning site. Cell transfection was performed with PolyJET (SignaGen, Gaithersburg, MD, USA) as described in the manufacturer's protocol. The viruses were propagated in 293T cells by cotransfecting the corresponding plasmids with the helper plasmids.

Establishment of the stable cell lines. The formation of the stable cell lines was previously described (24). For stable overexpression of Siah1, the U251 cells were infected by GFP or GFP-Siah1 viruses, respectively. Forty-eight hours after infection, the cells were continuously cultured in medium containing $2.5 \mu \mathrm{g} / \mathrm{ml}$ puromycin (Sigma, St. Louis, MO, USA). The surviving cells were cultured into cell lines stably expressing GFP or GFP-Siah1. For the silencing of Siah1, the scramble or shSiah1 virus-infected U251 cells were subjected to the sorting of GFP-positive cells by flow cytometry. GFP-positive cells were cultured to produce stable lines silenced for Siah1.

Western blotting. Equal amounts of protein lysates were subjected to $10 \%$ SDS-PAGE and then transferred to a PVDF
A
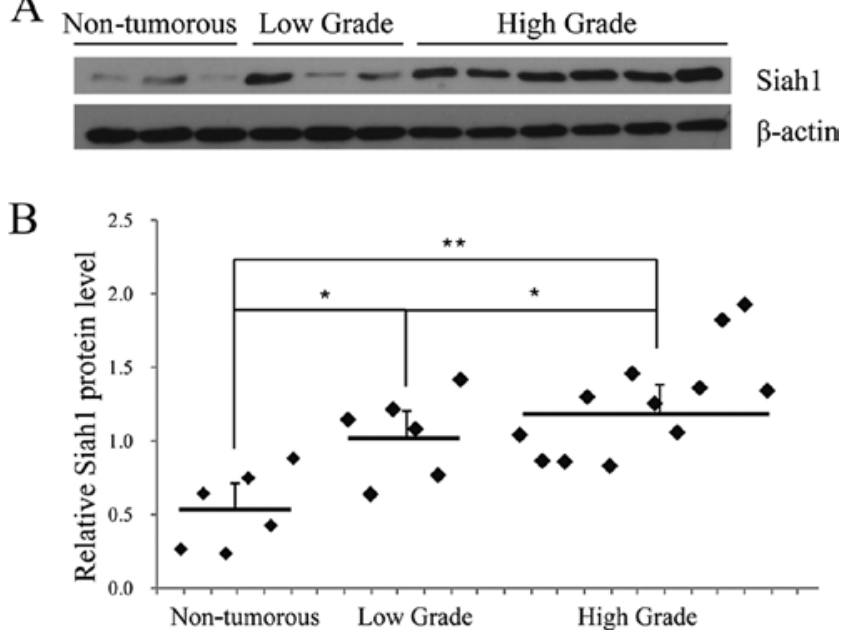

Figure 1. Siah1 expression is increased in human glioma tissues. Total proteins isolated from non-tumorous brain and glioma tissues were subjected to western blotting for assessment of Siah1. (A) Representative blots of Siah1 from non-tumorous brain and human glioma tissues, $\beta$-actin served as an internal control. (B) Statistical analysis of the relative level of Siah1 in nontumorous brain tissues $(n=6)$, low-grade glioma tissues $(n=6)$ and high-grade glioma tissues $(\mathrm{n}=12),{ }^{*} \mathrm{P}<0.05,{ }^{* *} \mathrm{P}<0.01$.

membrane (Millipore, Billerica, MA, USA) and probed with the primary antibodies (Siah1, PHD3, HIF-1 $\alpha, \beta$-actin) at $4{ }^{\circ} \mathrm{C}$ overnight and the secondary antibodies at room temperature for $1 \mathrm{~h}$. Bound antibodies were detected by the ECL Plus Western Blotting Substrate (Thermo Fisher, Waltham, MA, USA) and exposed to X-ray film. Band densities were quantified by ImageJ software. The relative amount of the proteins was determined by normalization of the densitometry.

Wound healing assay. The stable cell lines were cultured in a 6-well plate under normal conditions for $24 \mathrm{~h}$ and starved by no serum for $12 \mathrm{~h}$. Then, scratches were performed in the middle of the wells with a pipette tip. Thereafter, the cells were cultured under a hypoxic condition $\left(5 \% \mathrm{CO}_{2}, 1 \% \mathrm{O}_{2}\right.$ and $94 \%$ $\mathrm{N}_{2}$ ) for 12 and $24 \mathrm{~h}$. The images were captured by an inverted microscope (IX71; Olympus, Tokyo, Japan) at the designated time points. The number of cells crossing the wound was normalized according to the control.

Invasion assay. Cell invasion was assessed using Matrigelprecoated Transwell inserts $(8.0-\mu \mathrm{m}$ pore size with polyethylene tetraphthalate membrane; Invitrogen) according to the manufacturer's protocol. The pretreated cell suspension $\left(1 \times 10^{5}\right)$ in serum-free culture media was added into the inserts, and each insert was placed in the lower chamber filled with culture media containing $10 \%$ FBS as a chemoattractant. The invasion chambers were incubated under a hypoxic condition for $24 \mathrm{~h}$. Then, the non-invasive cells were removed from the upper chamber; filters were fixed with methanol for $15 \mathrm{~min}$ and stained with a $0.1 \%$ crystal violet solution for $10 \mathrm{~min}$. Five fields of adherent cells in each well were randomly photographed under an inverted microscope and counted.

Statistical analysis. Data are presented as the means \pm SEM. Statistical significance was determined using the Student's 
A

B


C

Scramble

shSiah1B

$\mathrm{Oh}$
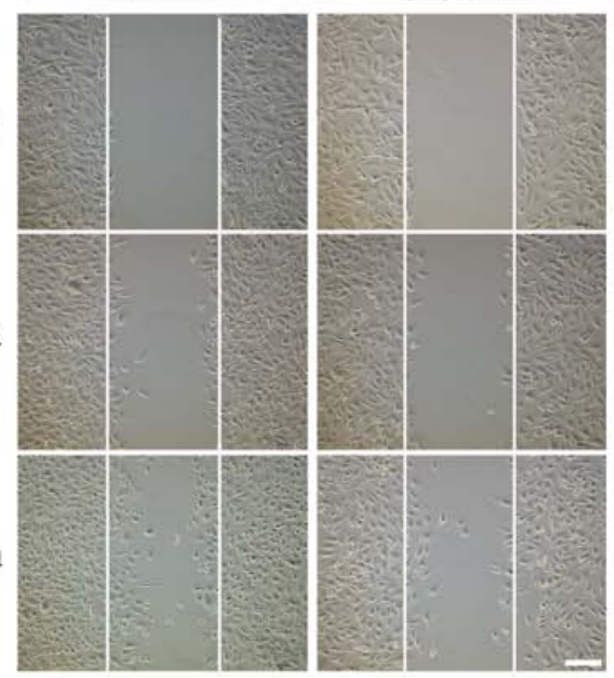

Scramble

shSiah1B

$12 \mathrm{~h}$

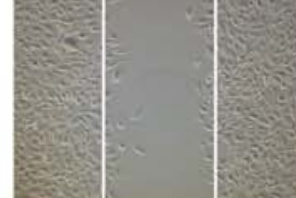

$24 \mathrm{~h}$



Bright field

D

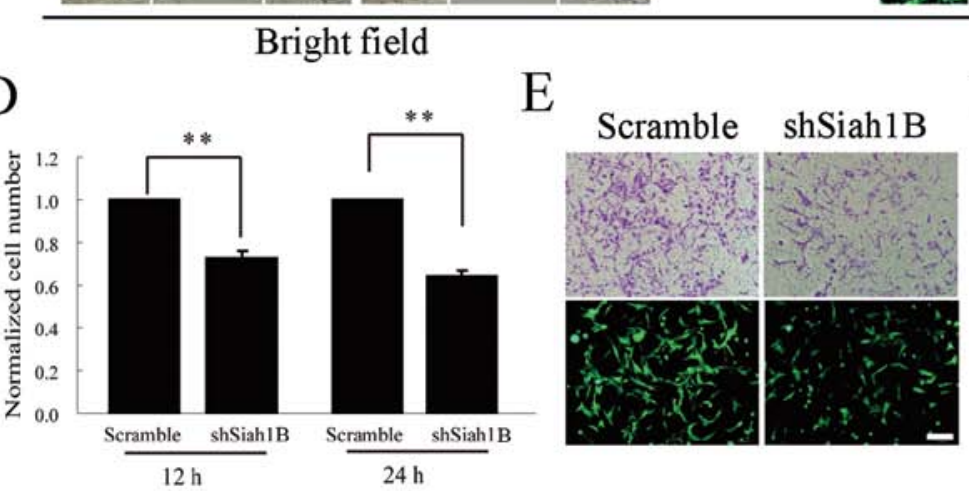

$\mathrm{Oh}$


$12 \mathrm{~h}$
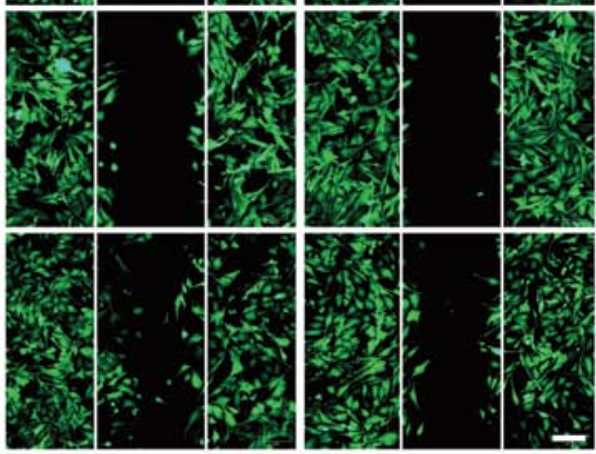

$24 \mathrm{~h}$

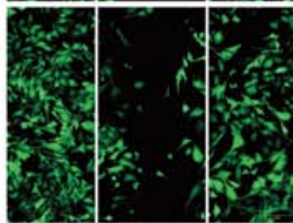

Fluorescence field

F

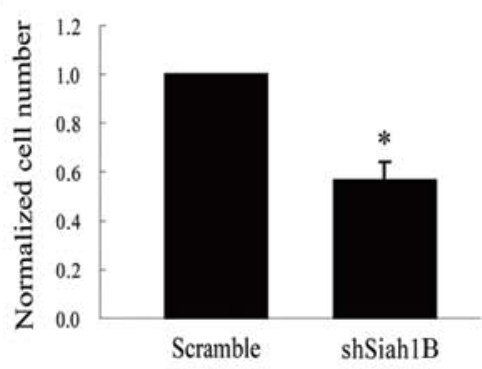

Figure 2. Downregulation of Siah1 inhibits glioma cell migration and invasion under hypoxia. U251 cells stably expressing the scramble or shSiah1 were subjected to a wound healing assay for migration and a Transwell assay for assessment of invasion. (A) Silencing efficiency of Siah1 shRNAs was examined by western blotting. (B) Fluorescence and western blotting confirmed that the stable cell lines expressed the scramble or shSiah1. (C and D) Wound healing assay showed that inhibition of Siah1 reduced glioma cell migration compared with the control. Scale bar, $100 \mu \mathrm{m} .{ }^{* *} \mathrm{P}<0.01$. (E and F) Silencing of Siah1 decreased glioma cell invasion. Scale bar, $100 \mu \mathrm{m} .{ }^{~} \mathrm{P}<0.05$.

t-test, and $\mathrm{P}<0.05$ was considered to indicate a statistically significant result.

\section{Results}

Siahl is expressed increasingly in human glioma tissues. In order to understand the possible role of Siah1 in human glioma, we examined the Siah1 expression in 6 specimens of non-tumorous brain tissues and 18 specimens of glioma tissues ( 6 low-grade and 12 high-grade) by western blotting. A representative blot is shown in Fig. 1A. The statistical results indicated that the protein level of Siah1 in the glioma tissues was higher than that in the non-tumorous tissues and was correlated with advanced tumor status and stage (Fig. 1A and B). These results suggest that Siah1 expression is upregulated in human glioma, which provides us with initial evidence that Siah1 plays a role in the development and progression of human glioma.

Downregulation of Siahl inhibits glioma cell migration and invasion under hypoxia. To investigate the possible role of Siah1 in the development and progression of human glioma, we utilized loss-of- and gain-of function approaches. Firstly, we downregulated Siah1 expression using its specific short 
A


$\mathrm{B}$

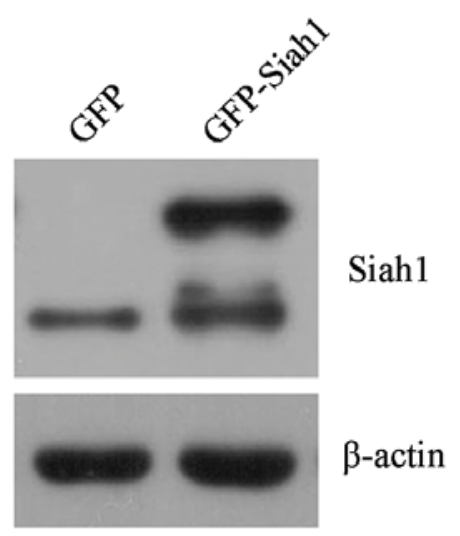

GFP-Siah1

$\mathrm{C}$

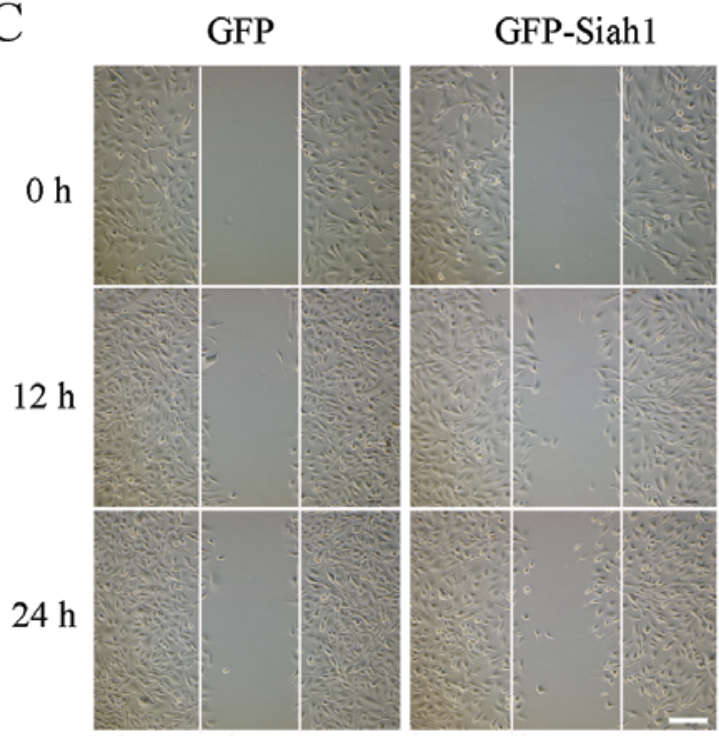

Bright field
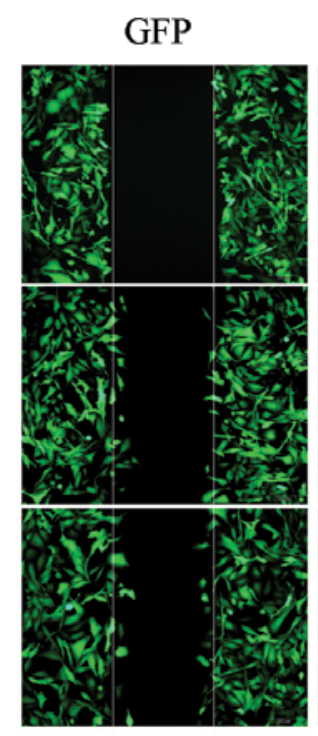

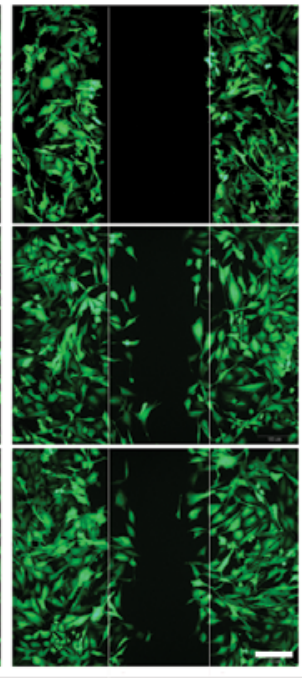

Fluorescence field

$\mathrm{D}$

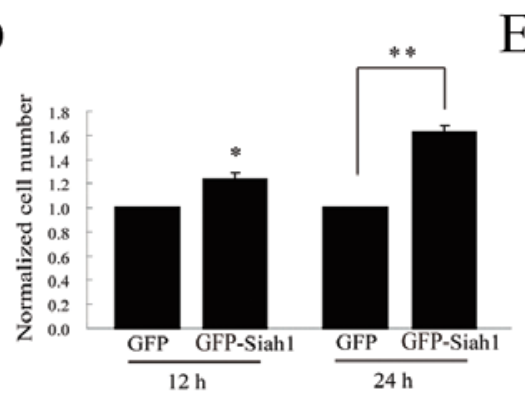

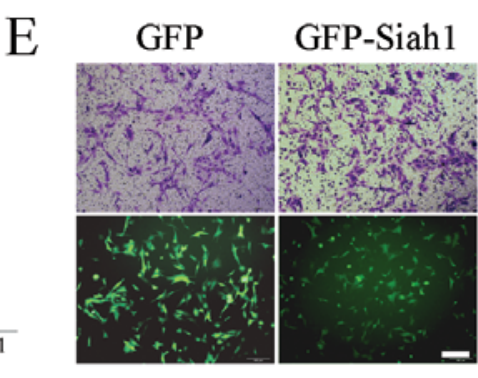

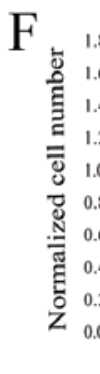

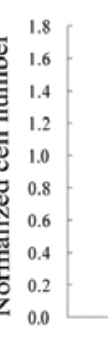

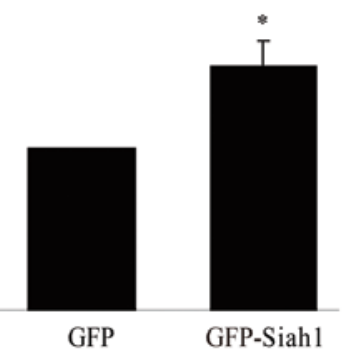

Figure 3. Overexpression of Siah1 promotes glioma cell migration and invasion under hypoxia. U251 cells stably expressing GFP or GFP-Siah1 were subjected to a wound healing assay for assessment of migration and a Transwell assay for assessment of invasion. (A) Stable cell lines expressing GFP or GFP-Siah1. (B) Confirmation of the expression by western blotting. (C and D) Wound healing assay showed that overexpression of Siah1 promoted glioma cell migration compared with the control. Scale bar, $100 \mu \mathrm{m} .{ }^{*} \mathrm{P}<0.05,{ }^{* *} \mathrm{P}<0.01$. (E and F) Overexpression of Siah1 reinforced glioma cell invasion. Scale bar, $100 \mu \mathrm{m}$. $\mathrm{P}<0.05$.

hairpin RNA and observed the effects on cell migration and invasion. For the silencing of Siah1, two shRNA targets (shSiah1A and shSiah1B) were cloned into the lentiviral vector pLL3.7 and screened for their efficacy in suppressing Siah1 expression, and a negative control shRNA (scramble) was used as a control. As shown in Fig. 2A, the silencing efficiency of shSiah1B was $\sim 80 \%$. Thereafter, shSiah1B and the scramble were packaged into the lentivirus in $293 \mathrm{~T}$ cells and used to establish the stable cell line with loss of Siah1 (Fig. 2B). Next, we investigated whether the cell migration and invasion were affected by the silencing of Siah1 under hypoxia in the stable cell lines. The wound healing assay showed that the number of migratory cells was reduced by 27 and $36 \%$ at 12 and $24 \mathrm{~h}$ (Fig. 2C and D). The Transwell assay showed that the invasive cells decreased by $43 \%$, compared with the corresponding controls (Fig. 2E and F). These results indicate 
A

\begin{tabular}{|c|c|c|c|c|c|c|c|c|}
\hline \multicolumn{4}{|c|}{ Scramble } & \multicolumn{4}{|c|}{ shSiah1B } & \\
\hline 0 & 1 & 3 & 5 & 0 & 1 & 3 & 5 & (h) \\
\hline 6 & $E$ & 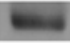 & $\omega$ & $=$ & - & $=$ & 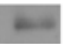 & HIF-1 $\alpha$ \\
\hline & $=$ & na & $=$ & & $=$ & & & PHD3 \\
\hline & & & & & & & & $\beta$-actin \\
\hline
\end{tabular}

$\mathrm{C}$


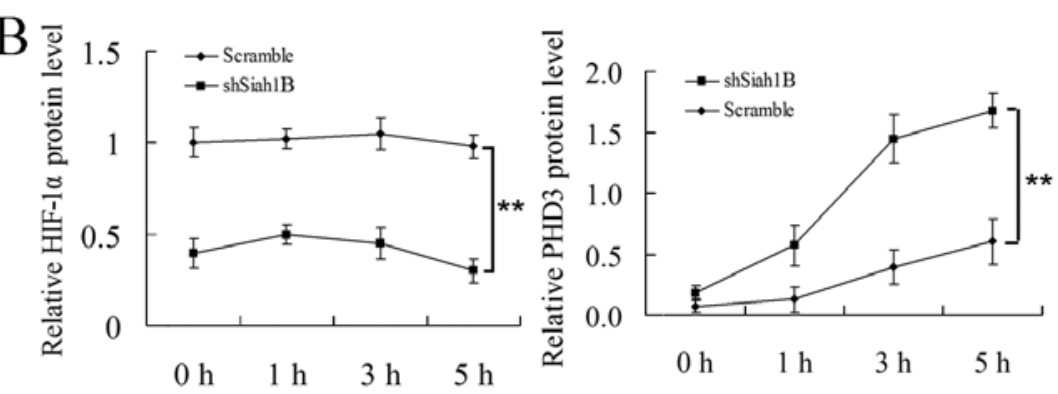

$\mathrm{D}$



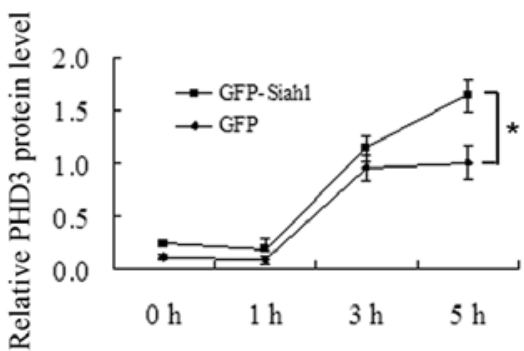

Figure 4. Siah1 induces HIF-1 $\alpha$ by promoting the degradation of PHD3 in human glioma cells under hypoxia. The cell lines with loss-of and gain-of Siah1 were treated under hypoxia. The cells were then harvested at the indicated time points and were lysed in lysis buffer. The lysates were subjected to western blotting for detection of HIF-1 $\alpha$ and PHD3. (A and C) Representative blots of PHD3 and HIF-1 $\alpha$. (B and D) Statistical analyses of the relative protein levels of HIF- $1 \alpha$ and PHD3. ${ }^{*} \mathrm{P}<0.05,{ }^{* *} \mathrm{P}<0.01$.

that Siah1 is involved in the migration and invasion of human glioma cells and downregulation of Siah1 inhibits the glioma cell migration and invasion under hypoxia.

Overexpression of Siahl promotes glioma cell migration and invasion under hypoxia. To further investigate the role of Siah1, we tested the effect of Siah1 on cell migration and invasion in U251 cells transfected by a lentivirus overexpressing Siah1. The stable cell lines overexpressing GFP or GFP-Siah1 were assessed by GFP imaging and western blotting (Fig. 3A and B). Then, we investigated whether the cell migration and invasion were promoted upon Siah1 overexpression under hypoxia in the stable lines expressing GFP or GFP-Siah1. The wound healing assay showed that the number of migratory cells was increased by 25 and $62 \%$ at 12 and $24 \mathrm{~h}$ (Fig. 3C and D) and the Transwell assay showed that the number of invasive cells increased by $51 \%$, when compared with the corresponding controls (Fig. 2E and F). These results further indicate that Siah1 is involved in the migration and invasion of human glioma cells, and overexpression of Siah1 promotes glioma cell migration and invasion under hypoxia.

Siahl induces HIF-1a by promoting the degradation of PHD3 in human glioma cells under hypoxia. It has been reported that HIF-1 $\alpha$ is activated under hypoxia and plays a crucial role in glioma cell migration and invasion (3); however, whether Siah1 is involved in the regulation of HIF- $1 \alpha$ in human glioma cells is still unclear. For this reason, we detected the expression level of PHD3 and HIF-1 $\alpha$ in Siah1 loss-of- and gain-of-function studies at several hypoxia time points by western blotting. We found that loss of Siah1 led to a more rapid increase in oxygen sensor PHD3 thereby inhibiting the production of HIF-1 $\alpha$ when compared with the control (Fig. 4A and B). In addition, we analyzed the role of Siah1 under the condition of overexpression. As shown in Fig. 4C and D, compared with the control, overexpression of Siah1 resulted in a slower increase in PHD3 thereby promoting the rapid production of HIF-1 $\alpha$. These results suggest that Siah1 promotes cell migration and invasion by regulating the degradation of PHD3 thereby improving the stability of HIF-1 $\alpha$.

\section{Discussion}

In the present study, we showed that Siah1 contributes to glioma cell migration and invasion by reducing the stability of PHD3, thereby stabilizing HIF-1 $\alpha$. The following results support our conclusion. Firstly, the western blotting results showed that Siah1 was upregulated in the human glioma tissues. Secondly, downregulation of Siah1 suppressed glioma cell migration and invasion while Siah1 overexpression promoted it. Thirdly, downregulation of Siah1 stabilized PHD3 and attenuated the production of HIF-1 $\alpha$ under hypoxia, while overexpression of Siah1 reduced the stability of PHD3 and accelerated the production of HIF-1 $\alpha$.

Hypoxia is one of the important features of human glioblastoma, which is associated with poor prognosis, increased angiogenesis, tumor growth and resistance to radiotherapy and chemotherapy (12). HIF-1 $\alpha$, a pivotal hypoxia regulatory factor, has been shown to promote both angiogenesis and invasion. Upregulation of HIF- $1 \alpha$ is an important physiological process of glioma adaptation to hypoxia (25). Our data showed that, when glioma cells were in a hypoxic condition, downregulation of Siah1 inhibited the production of HIF-1 $\alpha$ while overexpression of Siah1 promoted it. These data support that Siah1 plays an important role in the regulation of HIF-1 $\alpha$ under hypoxia and that inhibition of Siah1 may be an attractive therapeutic strategy by which to target the tumor microenvironment. 
Although the role of HIF-1 $\alpha$ in glioma cell progression has been well studied $(3,6,11-13)$, the regulatory mechanism to date remains unclear. It has been demonstrated that Rho small GTPases, Myc, mTOR and protein prenylation transferase GGTI are involved in regulating HIF-1 $\alpha$ induction (26-31). Particularly, it has been reported that ubiquitin ligase Siah2 contributes to the abundance of HIF-1 $\alpha$ by regulating PHD3 stability, thereby playing important roles in the cellular response to hypoxia (19). As Siah1 possesses a high similarity with Siah2, whether it plays an equivalent role under hypoxic conditions is still unknown. Our results indicate that Siah1 is involved in the regulation of HIF-1 $\alpha$ in human glioma and plays a similar role to that of Siah2.

In conclusion, the present study found that Siah1 is upregulated in human glioma tissues, and plays important roles in glioma cell migration and invasion in the cellular response to hypoxia. To our knowledge, this is the first study to focus on the role of Siah1 in glioma cells. The present study revealed the function of Siah1 in glioma cells and indicates that Siah1 may be a potential molecular target for the treatment of glioma based on the interference of the Siah1-PHD3-HIF-1 $\alpha$ signaling pathway.

\section{Acknowledgements}

This study was supported by the National Natural Science Foundation of China (nos. 81201264 and 81272777), and the China Postdoctoral Science Foundation.

\section{References}

1. Wen PY and Kesari S: Malignant gliomas in adults. N Engl J Med 5: 492-507, 2008.

2. Clarke J, Butowski N and Chang S: Recent advances in therapy for glioblastoma. Arch Neurol 67: 279-283, 2010.

3. Kaur B, Khwaja FW, Severson EA, et al: Hypoxia and the hypoxia-inducible-factor pathway in glioma growth and angiogenesis. Neuro Oncol 7: 134-153, 2005.

4. Vordermark D: Significance of hypoxia in malignant glioma. Re: Evans et al. Hypoxia is important in the biology and aggression of human glial brain tumors. Clin Cancer Res 11: 3966-3968, 2005.

5. Evans SM, Judy KD, Dunphy I, et al: Hypoxia is important in the biology and aggression of human glial brain tumors. Clin Cancer Res 10: 8177-8184, 2004

6. Kessler J, Hahnel A, Wichmann H, et al: HIF-1 $\alpha$ inhibition by siRNA or chetomin in human malignant glioma cells: effects on hypoxic radioresistance and monitoring via CA9 expression. BMC Cancer 10: 605, 2010.

7. Wilson WR and Hay MP: Targeting hypoxia in cancer therapy. Nat Rev Cancer 11: 393-410, 2011.

8. Lipkowitz S and Weissman AM: RINGs of good and evil: RING finger ubiquitin ligases at the crossroads of tumour suppression and oncogenesis. Nat Rev Cancer 11: 629-643, 2011.

9. Jans J, van Dijk JH, van Schelven S, et al: Expression and localization of hypoxia proteins in prostate cancer: prognostic implications after radical prostatectomy. Urology 75: 786-792, 2010.

10. Wong CC, Zhang H, Gilkes DM, et al: Inhibitors of hypoxiainducible factor 1 block breast cancer metastatic niche formation and lung metastasis. J Mol Med 90: 803-815, 2012.
11. Dai S, Huang ML, Hsu CY and Chao KS: Inhibition of hypoxia inducible factor $1 \alpha$ causes oxygen-independent cytotoxicity and induces p53 independent apoptosis in glioblastoma cells. Int J Radiat Oncol Biol Phys 55: 1027-1036, 2003.

12. Lu H, Li Y, Shu M, et al: Hypoxia-inducible factor-1 $\alpha$ blocks differentiation of malignant gliomas. FEBS J 276: 7291-7304, 2009.

13. Gillespie DL, Flynn JR, Ragel BT, et al: Silencing of HIF-1a by RNA interference in human glioma cells in vitro and in vivo. Methods Mol Biol 487: 283-301, 2009.

14. Carthew RW and Rubin GM: seven in absentia, a gene required for specification of R7 cell fate in the Drosophila eye. Cell 63: 561-577, 1990.

15. Hu G, Chung YL, Glover T, et al: Characterization of human homologs of the Drosophila seven in absentia (sina) gene. Genomics 46: 103-111, 1997.

16. Habelhah H, Frew IJ, Laine A, et al: Stress-induced decrease in TRAF2 stability is mediated by Siah2. EMBO J 21: 5756-5765, 2002.

17. Habelhah H, Laine A, Erdjument-Bromage H, et al: Regulation of 2-oxoglutarate ( $\alpha$-ketoglutarate) dehydrogenase stability by the RING finger ubiquitin ligase Siah. J Biol Chem 279: 53782-53788, 2004.

18. Matsuzawa SI and Reed JC: Siah-1, SIP, and Ebi collaborate in a novel pathway for $\beta$-catenin degradation linked to $\mathrm{p} 53$ responses. Mol Cell 7: 915-926, 2001.

19. Nakayama K, Frew IJ, Hagensen M, et al: Siah2 regulates stability of prolyl-hydroxylases, controls HIF1 $\alpha$ abundance, and modulates physiological responses to hypoxia. Cell 117: 941-952, 2004.

20. Zhang J, Guenther MG, Carthew RW and Lazar MA: Proteasomal regulation of nuclear receptor corepressor-mediated repression. Genes Dev 12: 1775-1780, 1998.

21. Matsuo K, Satoh S, Okabe H, et al: SIAHI inactivation correlates with tumor progression in hepatocellular carcinomas. Genes Chromosomes Cancer 36: 283-291, 2003.

22. Wen YY, Yang ZQ, Song M, et al: The expression of SIAH1 is downregulated and associated with Bim and apoptosis in human breast cancer tissues and cells. Mol Carcinog 49: 440-449, 2010.

23. Kitagawa N, Kondo S, Wakisaka N, et al: Expression of sevenin-absentia homologue 1 and hypoxia-inducible factor 1 alpha: novel prognostic factors of nasopharyngeal carcinoma. Cancer Lett 331: 52-57, 2013.

24. Shi H, Gao Y, Tang Y, et al: CacyBP/SIP protein is important for the proliferation of human glioma cells. IUBMB Life 66: 286-291, 2014.

25. Liu Y, Li YM, Tian RF, et al: The expression and significance of HIF- $1 \alpha$ and GLUT-3 in glioma. Brain Res 1304: 149-154, 2009.

26. Zhou J, Li K, Gu Y, et al: Transcriptional up-regulation of RhoE by hypoxia-inducible factor (HIF)-1 promotes epithelial to mesenchymal transition of gastric cancer cells during hypoxia. Biochem Biophys Res Commun 415: 348-354, 2011.

27. Xue Y, Li NL, Yang JY, et al: Phosphatidylinositol 3'-kinase signaling pathway is essential for Rac1-induced hypoxia-inducible factor- $1 \alpha$ and vascular endothelial growth factor expression. Am J Physiol Heart Circ Physiol 300: H2169-H2176, 2011.

28. Zhang P, Zhang X, Hao X, et al: Racl activates HIF-1 in retinal pigment epithelium cells under hypoxia. Graefes Arch Clin Exp Ophthalmol 247: 633-639, 2009.

29. Kim JW, Gao P, Liu YC, Semenza GL and Dang CV: Hypoxiainducible factor 1 and dysregulated c-Myc cooperatively induce vascular endothelial growth factor and metabolic switches hexokinase 2 and pyruvate dehydrogenase kinase 1. Mol Cell Biol 27: 7381-7393, 2007.

30. Yuan G, Nanduri J, Khan S, et al: Induction of HIF-1 $\alpha$ expression by intermittent hypoxia: involvement of NADPH oxidase, $\mathrm{Ca}^{2+}$ signaling, prolyl hydroxylases, and mTOR. J Cell Physiol 217: 674-685, 2008.

31. Zhou X, Liu Z, Shi Q, et al: Geranylgeranyltransferase I regulates HIF-1 $\alpha$ promoting glioblastoma cell migration and invasion. J Neurooncol 112: 365-374, 2013. 